\title{
Calibration of bridge standard for strain gauge bridge amplifier
}

\author{
David Corminboeuf ${ }^{\mathrm{a}}$ \\ Federal Institute of Metrology METAS, Lindenweg 50, 3003 Bern-Wabern, Switzerland
}

\begin{abstract}
This paper presents an automatic system developed at METAS for the calibration of bridge standards used as reference for strain gauge bridge amplifiers. This system, based on a comparison method, uses a PXI chassis with two acquisition boards to balance the bridge automatically. The use of two specific inductive voltage dividers is required to reach the very high accuracy of this type of bridge standard. With a ratio of $2 \mathrm{mV} / \mathrm{V}$, an input voltage of $10 \mathrm{~V}$ at $225 \mathrm{~Hz}$, the relative uncertainty is $8 \mathrm{ppm}$, which is $16 \mathrm{ppb}$ of the input voltage.
\end{abstract}

\section{Introduction}

The strain gauge bridges are routinely used in measurements of force, weight, torque, pressure. A sensor is used to convert a mechanical signal into an electrical signal. The electric signal must be amplified, conditioned and measured before a value to the user is returned. All these operations are usually carried out within a single instrument, a strain gauge bridge amplifier. To calibrate a strain gauge sensor, it is first necessary to calibrate the amplifier by using a standard bridge. This bridge standard is an inductive voltage divider (IVD) that simulates a strain gauge bridge with the additional advantage of providing a voltage ratio with a greater accuracy and a better stability. The better the uncertainty of this voltage ratio, the better the accuracy of the whole measurement chain. The goal of this paper is to present the measurement system developed at METAS for the calibration of this type of bridge standards.

\section{The strain gauges}

\subsection{Principle}

A strain gauge is a metal alloy track mounted on a very thin plastic foil. This backing allows the strain gauge to be handled and it also serves as electrical insulation between the metal foil and the piece whose deformation must be measured. This deformation will cause a tension or compression of the gauge which will result in a variation in its electrical resistance [1].

\subsection{Topology}

\footnotetext{
${ }^{\mathrm{a}}$ Corresponding author: david.corminboeuf@metas.ch
}

The most accurate way to measure this very small change in resistance is by using a Wheatstone bridge (Figure 1). This bridge is designed to measure a relative change but can be found in three variants, the $1 / 4$ bridge, the $1 / 2$ bridge and the full bridge containing respectively 1,2 and 4 gauges. The full bridge has the best accuracy because it has a greater sensitivity when the gauges are placed in opposition, two in tension and two in compression: the effects add up. Another advantage is the immunity to temperature variations, these variations are compensated because they are the same in each branch of the bridge.

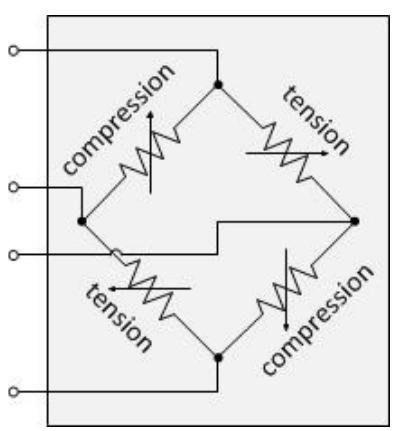

Figure 1. Full Wheatstone bridge

\subsection{Measurement system}

Many commercial measurement systems for strain gage sensors exist. The measurement system consists mainly of a voltage source used to supply the bridge, an amplifier to amplify the very small output signal of the bridge, a signal conditioning and processing unit and a display to give the result of the ratio $U_{O U T} / U_{I N}$ in $\mathrm{mV} / \mathrm{V}$ [2].

\subsection{Traceability}


To calibrate a strain gauge sensor, a reference measuring system is needed. The calibration of the measuring system is performed by using a standard bridge. There are different types of standard bridge, but the most accurate are based on inductive voltage dividers like the HBM BN100 (Figure 2). It has two differential outputs, on each output and between inputs, $350 \Omega$ resistors has been added to simulate the resistance of a strain gauge sensor. This instrument can provide a ratio voltage of $-100 \mathrm{mV} / \mathrm{V}$ to $100 \mathrm{mV} / \mathrm{V}$ with steps of $0.1 \mathrm{mV} / \mathrm{V}$ and a measurement uncertainty of $10 \mathrm{ppm}$ at $2 \mathrm{mV} / \mathrm{V}$, which is an absolute uncertainty of $20 \mathrm{ppb}$ [3]. The calibration of this instrument is described in the following sections.

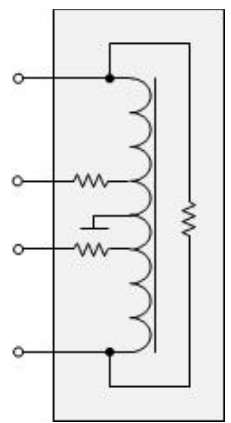

Figure 2. Bridge standard

\section{Calibration method}

The measuring principle is similar to the one of a bridge for inductive voltage divider (IVD) calibration [4] using a comparison method (Figure 3). The two dividers are powered in parallel and the comparison between the output voltage of the reference divider and that of the UUT allows the determination of the error. The balancing of the bridge is done by using a detection and an injection transformer located between the two outputs. These transformers have a ratio of 1 to 100 . The traceability of this measurement is ensured by the fact that METAS is primary in IVD calibration.

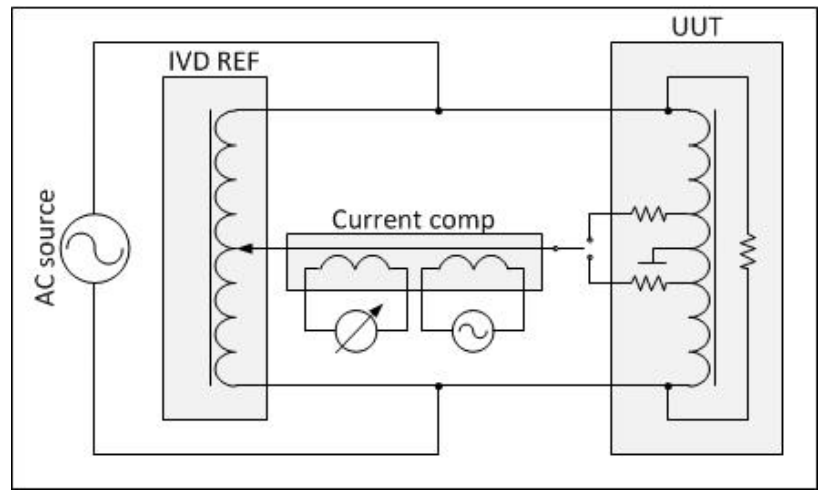

Figure 3. Schematic of the measuring principle

The calibration of the bridge standard shows three main differences with respect to the calibration of an IVD described previously. Firstly, the bridge standard has two differential outputs referenced to a zero volt at the centre. The output of an IVD is referenced to the input low. Secondly, the required precision level of $10 \mathrm{ppm}$ at 2 $\mathrm{mV} / \mathrm{V}$ is better than what we can reach with the available
IVD calibration system. Thirdly, the output impedance of $350 \Omega$ decreases the sensitivity of the detector.

To solve the first problem an isolation transformer is used to supply the IVD and the bridge standard to have a symmetrical power supply instead of a signal referenced to the low side.

The measurement of the differential output of the bridge standard must be done by two successive measurements referenced to the zero given by the bridge. When the two measurements have the same uncertainties this increases the uncertainty as follow:

$$
u_{\text {diff }}=\sqrt{u_{\text {meas } 1}^{2}+u_{\text {meas } 2}^{2}}=u_{\text {meas }} \cdot \sqrt{2}
$$

The best uncertainty of a voltage ratio we can reach with a reference IVD at $225 \mathrm{~Hz}$ is $60 \mathrm{ppb}$. In differential mode, it is $90 \mathrm{ppb}$ (equation (1)). The goal of this calibration is an uncertainty under $20 \mathrm{ppb}$.

The equation for only one of the two differential outputs when the bridge is balanced is:

$$
U_{S} \cdot\left(D_{R}+\delta_{R}\right)=U_{S} \cdot\left(D_{U}+\delta_{U}\right)+U_{i n j}
$$

With:

$$
\begin{array}{ll}
U_{S}: & \text { source voltage } \\
D_{R}: & \text { ratio of IVD REF } \\
D_{U}: & \text { ratio of UUT } \\
\delta_{R}: & \text { ratio error of IVD REF } \\
\delta_{U}: & \text { ratio error of UUT } \\
U_{\text {inj }}: & \text { injection voltage }
\end{array}
$$

The solution of the second problem is to develop a dedicated divider mounted in series with the reference divider [5]. Substituting IVD REF by two IVDs in series, IVD1 and IVD2, the new equation of the balanced bridge is:

$$
\left(D_{1}+\delta_{1}\right) \cdot\left(D_{2}+\delta_{2}\right)=\left(D_{U}+\delta_{U}\right)+\frac{U_{\text {inj }}}{U_{S}}
$$

The total ratio without considering the errors is:

$$
D_{\text {tot }}=D_{1} \cdot D_{2}
$$

And the total uncertainty:

$$
u_{t o t}=\sqrt{\left(u_{1}\right)^{2}+\left(u_{2}\right)^{2}}
$$

With:

$$
\begin{aligned}
& u_{1}=u \cdot D_{2} \\
& u_{2}=u \cdot D_{1}
\end{aligned}
$$

Where:

$$
\begin{aligned}
& D_{1} \text { is the ratio of IVD1 } \\
& D_{2} \text { is the ratio of IVD2 } \\
& u \text { is the uncertainty of IVD1 and IVD2 }
\end{aligned}
$$

From (5) with (6) and (7), $u_{\text {tot }}$ becomes:

$$
u_{\text {tot }}=\sqrt{\left(u \cdot D_{2}\right)^{2}+\left(u \cdot D_{1}\right)^{2}}=u \cdot \sqrt{D_{2}{ }^{2}+D_{1}{ }^{2}}
$$


Because the ratio of $2 \mathrm{mV} / \mathrm{V}$ is a common ratio for many strain gauge sensors, the new divider has been dimensioned to have the best uncertainty for this ratio.

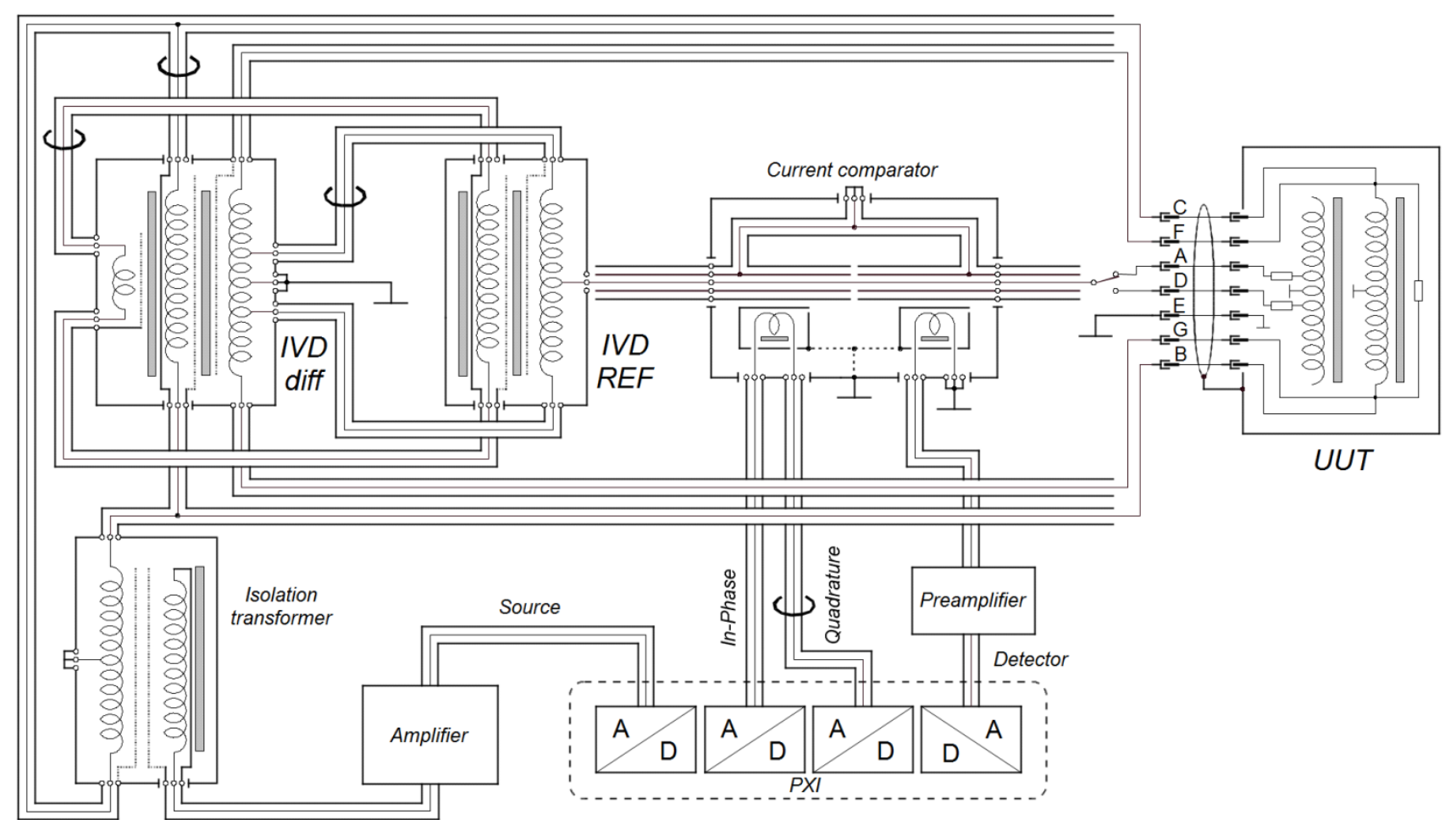

Figure 4. Schematic of the bridge to calibrate the bridge standard

Equation (8) has a minimum with $D_{1}=D_{2}$. The ratio of the new divider, named IVD $_{\text {diff }}$, can be calculated for a total ratio of $2 \frac{\mathrm{mV}}{\mathrm{V}}=\frac{1}{500}$ with equation (4):

$$
\begin{gathered}
D_{\text {tot }}=D_{1} \cdot D_{2}=D^{2}=\frac{1}{500} \\
D=\sqrt{\frac{1}{500}}=\frac{1}{22.36}
\end{gathered}
$$

If we choose $D_{1}=\frac{1}{25}$ and $D_{2}=\frac{1}{20}$ the total uncertainty becomes:

$$
u_{t o t}=u \cdot \sqrt{\left(\frac{1}{25}\right)^{2}+\left(\frac{1}{20}\right)^{2}}=\frac{u}{15.6}
$$

About the third problem, the sensitivity of the detector can be increased by adding a low noise preamplifier at the output of the current comparator.

The detailed schematic of the system is presented in Figure 4.

\section{System description}

This section provides a more detailed explanation of the components of this bridge (Figure 4). The divider $\mathrm{IVD}_{\text {diff }}$ and the current comparator have been developed inhouse, the rest are commercial devices. All cables used in the bridge are coaxial except two. The cables linking the outputs of the UUT and IVD $\mathrm{REF}_{\text {, }}$ which are triaxial, but the two shields are connected together because the active guard is not necessary due to the very low voltage levels. The cable connected to the UUT is a 7 wires shielded cable, 3 meters long, which is an integral part of the UUT.

\subsection{Sampling system}

The sampling system is composed of a PXI chassis and two boards type NI-PXI4461 from National Instruments [4]. These two boards contain two 24 bit ADCs and two 24 bit DACs each. The maximum sampling rate is 204.8 $\mathrm{kS} / \mathrm{s}$ (kilo samples per second) and the maximum peak amplitude is $\pm 10 \mathrm{~V}$. This system is controlled by a computer connected via fiber optic. The software developed in LabVIEW is used to automatically balance the bridge.

\subsection{IVD diff}

The differential IVD is a transformer developed at METAS to reduce the uncertainty of the reference voltage ratio. It is mounted before IVD $\mathrm{REF}_{\text {, }}$, so it must have two outputs, one for the magnetising winding and one for the ratio winding and to be stable during the time of the measurement.

It is a two stages, single shielded transformer which consists of two windings wound around two magnetic cores. The first winding (magnetising winding) is wound around the first core and the second winding (ratio winding) is wound around the two cores. These two 
windings have a copper overlaying electrical screen to avoid parasitic effects between the primary and secondary windings. The two secondary windings are separately shielded to avoid parasitic effects on ratio. Each of the four windings is made with an annular wire back around the core to reduce sensitivity to external magnetic fields. This transformer has a 25 to 1 ratio with 250 turns for the primary winding and 10 turns for the secondary winding and works at a frequency of $225 \mathrm{~Hz}$ and a maximum voltage of $25 \mathrm{~V}$.

\subsection{IVD}

The reference divider is a two stages, single shielded divider with eight decades. It works at frequencies from $40 \mathrm{~Hz}$ to $10 \mathrm{kHz}$ and a maximum rating of $0.07 \mathrm{~V} / \mathrm{Hz}(15$ $\mathrm{V}$ at $225 \mathrm{~Hz}$ ).

\subsection{Isolation transformer}

It is a one stage, double shielded transformer. It has a 1 to 1 ratio and works at frequencies from $40 \mathrm{~Hz}$ to $10 \mathrm{kHz}$ and a maximum rating of $0.07 \mathrm{~V} / \mathrm{Hz}(15 \mathrm{~V}$ at $225 \mathrm{~Hz})$.

\subsection{Current comparator}

The main purpose of the current comparator is to detect and balance the small voltage difference between the output of the UUT and the output of the reference IVD. If the phase and/or the amplitude of the voltages applied to the current comparator are not equal, a small current will flow along the central conductor of the triaxial cable shown in Figure 4. This current will then be detected using a first 1 to 100 transformer. To balance this residual current, a small voltage (adjusted in amplitude and in phase) is injected in the central conductor using a second 1 to 100 transformer.

\subsection{Preamplifier}

It is a low noise preamplifier with $1.5 \mathrm{nV} / \mathrm{Hz}^{1 / 2}$ at $1 \mathrm{kHz}$. Its input impedance is $3.3 \mathrm{G} \Omega$ in parallel with $100 \mathrm{pF}$ and it is battery powered. No special accuracy is required for this amplifier because it amplifies the signal for the null detector at $225 \mathrm{~Hz}$.

\section{Measurements}

Between June and October 2014, measurements have been carried out at METAS with this new bridge on the bridge standard used as reference device by our "Mass, Force and Pressure" laboratory.

Three series of measurement have covered the range from $0.0 \mathrm{mV} / \mathrm{V}$ to $2.0 \mathrm{mV} / \mathrm{V}$. Each measurement is an average of 100 measurement points for the two differential outputs. The error represented in Figure 5 corresponds only to the in-phase error because the quadrature error is never used and measured by the strain gauge amplifiers.
These results are compared with those obtained by the calibration of the same bridge standard at the PTB in October 2014.

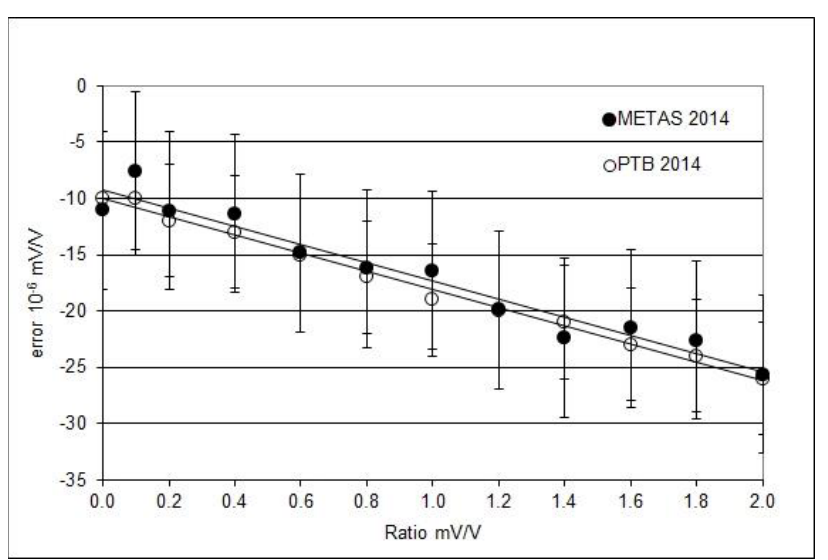

Figure 5. Comparison of the measurement results from METAS and PTB about the error of a bridge standard for the ratio 0.0 $\mathrm{mV} / \mathrm{V}$ to $2.0 \mathrm{mV} / \mathrm{V}$

The results obtained by METAS and the PTB are in very good agreement. The difference e.g. at $2 \mathrm{mV} / \mathrm{V}$ is less than $1 \mathrm{ppb}$. These good results validate our new measurement system.

Another range was also measured from $0 \mathrm{mV} / \mathrm{V}$ to 9 $\mathrm{mV} / \mathrm{V}$ which is the second decade of the bridge standard. Only one series of 100 measurements was carried out and the results are presented in Figure 6.

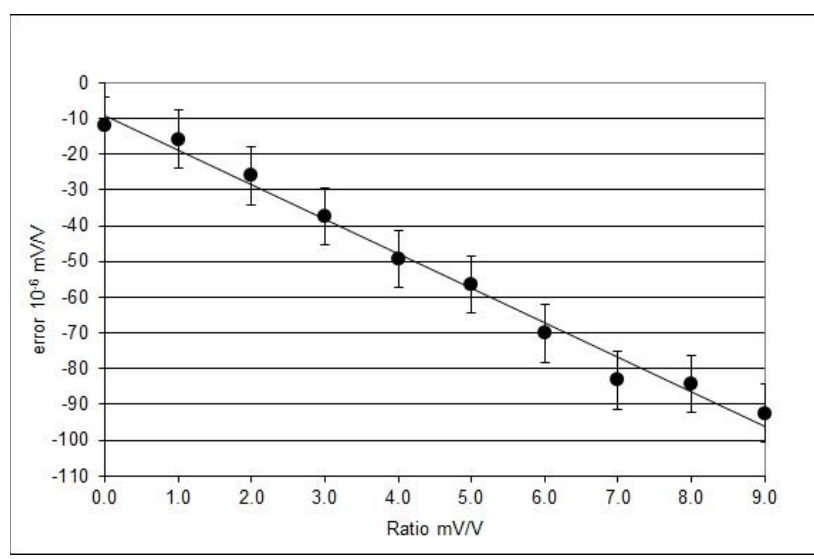

Figure 6. Measurement results of the error of a bridge standard for the ratio $0 \mathrm{mV} / \mathrm{V}$ to $9 \mathrm{mV} / \mathrm{V}$

\section{Uncertainty components}

As developed in chapter 3, the total uncertainty of the two IVDs can be calculated as follow:

With an uncertainty of $30 \mathrm{ppb}$ for each IVD

$u_{I V D S}=\frac{30}{15.6} \cdot \sqrt{2}=2.7 \mathrm{ppb}$

The uncertainty of the current comparator at $225 \mathrm{~Hz}$ is $u_{c c}=0.2 \mathrm{ppb}$

To evaluate the uncertainty of the injection linearity, two measurement series were done; one by balancing the bridge automatically, the other by balancing the bridge 
manually with $\mathrm{IVD}_{\mathrm{REF}}$ and the measured difference was less than $1 \mathrm{ppb}$.

The uncertainty due to the noise is based on the 100 measurements of each series with the ratios between 0.0 and $2.0 \mathrm{mV} / \mathrm{V}$. The standard deviation was $7 \mathrm{ppb}$.

The uncertainty due to the reproducibility is based on the 3 measurement series and the maximum standard deviation was $2.6 \mathrm{ppb}$.

\begin{tabular}{|l|c|c|}
\cline { 2 - 3 } \multicolumn{1}{c|}{} & Type & $u\left(10^{-9}\right)$ \\
\hline Two IVD in series & B & 2.7 \\
\hline Current Comparator & B & 0.2 \\
\hline Injection linearity & B & 1 \\
\hline Measured noise & A & 7 \\
\hline Repeatability & A & 2.6 \\
\hline $\boldsymbol{U}(\boldsymbol{k}=\mathbf{2})$ & & $\mathbf{1 6}$ \\
\cline { 3 - 3 } & &
\end{tabular}

Table 1. Uncertainty budget

\section{Conclusion}

Presently, this new measurement capability enables a calibration of a bridge standard type BN100 within its specifications for the three ratio decades. For example, for a ratio of $2 \mathrm{mV} / \mathrm{V}$, the relative uncertainty is $8 \mathrm{ppm}$ which corresponds to an uncertainty of $16 \mathrm{ppb}(\mathrm{k}=2)$ relative to the input.

The uncertainty due to the noise can be reduced. Improvement of the shielding should reduce this uncertainty.

The two successive measurements of the differential output are time-consuming. A modification to carry out the differential measurement in one phase is actually in progress.

\section{References}

1. Karl Hoffmann, An Introduction to Stress Analysis and Transducer Design using Strain Gauges, (HBM 2012), available at http://www.hbm.com

2. HBM, Operating manual Digital precision measuring amplifier DMP40, DMP40S2

3. HBM, Operating manual Bridge Calibration Unit for $225 \mathrm{~Hz} B N 100 \mathrm{~A}$

4. D. Corminboeuf and F. Overney, Inductive voltage divider calibration with sampling method, EPJ Web of Conferences 77, 00014 (2014), available at http://dx.doi.org/10.1051/epjconf/20147700014

5. G. Ramm, Calibration of bridge standards for use in strain-gage measurements, Reports in Applied Measurement RAM, Vol. 6, S. 26-30, (1990) 